\title{
CHARACTERIZING WHICH POWERS OF HYPERCUBES AND FOLDED HYPERCUBES ARE DIVISOR GRAPHS
}

\author{
Eman A. AbuHijleh ${ }^{1}$, Omar A. AbuGhneim² \\ AND \\ HASAN AL-EzEH ${ }^{2}$ \\ ${ }^{1}$ Department of Basic Sciences \\ Al-Zarka University College \\ Al-Balqa' Applied University \\ Zarqa 313, Jordan \\ ${ }^{2}$ Departments of Mathematics \\ Faculty of Science \\ The University of Jordan \\ Amman 11942, Jordan \\ e-mail: emanhijleh@bau.edu.jo \\ o.abughneim@ju.edu.jo \\ alezehh@ju.edu.jo
}

\begin{abstract}
In this paper, we show that $Q_{n}^{k}$ is a divisor graph, for $n=2$, 3. For $n \geq 4$, we show that $Q_{n}^{k}$ is a divisor graph iff $k \geq n-1$. For folded-hypercube, we get $F Q_{n}$ is a divisor graph when $n$ is odd. But, if $n \geq 4$ is even integer, then $F Q_{n}$ is not a divisor graph. For $n \geq 5$, we show that $\left(F Q_{n}\right)^{k}$ is not a divisor graph, where $2 \leq k \leq\left\lceil\frac{n}{2}\right\rceil-1$.
\end{abstract}

Keywords: hypercube, folded-hypercube, divisor graph, power of a graph. 2010 Mathematics Subject Classification: 05C12, 05C20, 05C65, 05C99.

\section{INTRODUCTION}

A graph $G$ is called a divisor graph if there is a bijection $f: V(G) \rightarrow S$, for some finite nonempty set $S$ of the positive integers, such that $u v \in E(G)$ if and only if $\operatorname{gcd}(f(u), f(v))=\min \{f(u), f(v)\}$ (this means $u v \in E(G)$ iff $f(u) \mid f(v)$ or $f(v) \mid f(u))$. The function $f$ is called a divisor labeling of $G$. 
Moreover, for a finite nonempty set $S$ of the positive integers, the divisor graph $G(S)$ of $S$ has $S$ as its vertex set and two distinct vertices $i$ and $j$ are adjacent if $i \mid j$ or $j \mid i$. A graph $G$ is a divisor graph if $G$ is isomorphic to $G(S)$. While the divisor digraph $D(S)$ of $S$ has a vertex set $S$ and $(i, j)$ is an arc of $D(S)$ iff $i$ divides $j$. In a digraph $D$, a transmitter is a vertex having indegree 0 , a receiver is a vertex having outdegree 0 , while a vertex $v$ is a transitive vertex if it has both positive outdegree and positive indegree, and $(u, w) \in E(D)$ whenever $(u, v) \in E(D)$ and $(v, w) \in E(D)$. An orientation $D$ of a graph $G$ in which every vertex is a transmitter, a receiver, or a transitive vertex is called a divisor orientation of $G$.

The length $g(n)$ of a longest path in the divisor graph whose divisor labeling has range $\{1,2, \ldots, n\}$ was studied in [8], [12], and [13]. The concept of a divisor graph involving finite nonempty sets of integers rather than positive integers was introduced in [14]. It was shown in [14] that odd cycles of length greater than three are not divisor graphs, while even cycles and caterpillars are. Indeed, not only caterpillars, but also all bipartite graphs are divisor graphs, as shown in [7]. Divisor graphs do not contain induced odd cycles of length greater than three, but they may contain triangles, see [7]. For instance, complete graphs are divisor graphs, see [7].

The distance between any two vertices $x$ and $y$ is the length of a shortest path between them. We denote this distance by $d_{G}(x, y)$. The diameter of a graph $G$ is equal to $\sup \left\{d_{G}(x, y): x, y \in V(G)\right\}$, denoted by $d_{G}$ or $\operatorname{diam}(G)$. The power graph of $G$ is denoted by $G^{k}$, where the vertex set of $G^{k}$ is $V(G)$ and two vertices $x, y$ are adjacent iff $d_{G}(x, y) \leq k$. A complete characterization of powers of paths, cycles and caterpillars that are divisor graphs were given in [1], [2], and [3].

More results on divisor graphs can be found in [4], [5], and [7]. For undefined notions and terminology, the reader is referred to [6].

\section{When a Power Graph of a Hypercube is a Divisor Graph}

In this section we characterize which powers of hypercubes are divisor graphs.

Definition. A hypercube $Q_{n}$ is a graph whose vertex set $V\left(Q_{n}\right)$ is the set of $n$-bit binary strings. (Any vertex of $Q_{n}$ may be labeled as $x=x_{1} x_{2} \cdots x_{n}$, where $x_{1} x_{2} \ldots x_{n}$ is the binary representation of $x$.) Two vertices $x=x_{1} x_{2} \cdots x_{n}$ and $y=y_{1} y_{2} \cdots y_{n}$ are adjacent if they differ in exactly one bit.

Alternatively, a hypercube can be defined recursively by $Q_{n}=Q_{n-1} \times K_{2}$, for $n \geq 2$ and $Q_{1}=K_{2}$. In Figure 1 , we have the graphs $Q_{1}, Q_{2}$, and $Q_{3}$.

It is well known that the diameter of $Q_{n}$ is $n$, hence $Q_{n}^{n}$ is a complete graph. Moreover, two vertices are adjacent in $Q_{n}^{k}$, if they differ in $k$ or less bits of their 


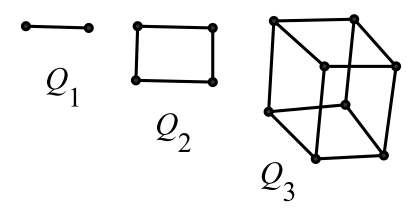

Figure 1. $Q_{1}, Q_{2}$, and $Q_{3}$.

binary strings.

The following important characterization of divisor graphs can be found in [7].

Theorem 1. Let $G$ be a graph. Then $G$ is a divisor graph if and only if $G$ has a divisor orientation. In particular, every bipartite graph is a divisor graph.

The following easy observation can be found in [7].

Proposition 2. Every induced subgraph of a divisor graph is a divisor graph.

We start with the following result for characterizing which powers of hypercubes are divisor graphs.

Theorem 3. Suppose that $Q_{n}$ is a hypercube. Then $Q_{n}^{n-1}$ is a divisor graph.

Proof. At first, two vertices in $Q_{n}^{n-1}$ are adjacent if they differ in $n-1$ or less bits of their binary strings. Then according to the labeling of vertices in $Q_{n}$, each vertex of $Q_{n}^{n-1}$ is adjacent to every other vertex of $Q_{n}^{n-1}$ except its complement in labeling. Hence, we can partition the vertices in $Q_{n}$ into $\left\{a_{i}: 1 \leq i \leq 2^{n-1}, i \in\right.$ $\mathbb{N}\} \cup\left\{b_{i}: 1 \leq i \leq 2^{n-1}, i \in \mathbb{N}\right\}$ such that, for each $i, a_{i}$ is the complement of $b_{i}$. Now, a divisor labeling $f$ of $Q_{n}^{n-1}$ is defined as follows:

$$
\begin{cases}f\left(a_{i}\right)=p^{i} q^{i-1} & \text { for } 1 \leq i \leq 2^{n-1}, i \in \mathbb{N} \\ f\left(b_{i}\right)=p^{i-1} q^{i} & \text { for } 1 \leq i \leq 2^{n-1}, i \in \mathbb{N}\end{cases}
$$

where $p$ and $q$ are distinct primes. Hence, $Q_{n}^{n-1}$ is a divisor graph.

Remark 4. This is an example of a graph for which both it and its complement graph $\left(\overline{Q_{n}^{n-1}}\right.$ being bipartite) are divisor graphs.

Note that $Q_{n}$ is a bipartite graph and hence a divisor graph. So, when $n=2$ or $3, Q_{n}^{k}$ is a divisor graph for any positive integer $k$. Now, we look at $n \geq 4$.

Lemma 5. $Q_{4}^{2}$ is not a divisor graph.

Proof. The induced subgraph on $\{0001,0010,0110,1111,1101\}$ in $Q_{4}^{2}$ is isomorphic to $C_{5}$. Hence, $Q_{4}^{2}$ is not a divisor graph by Proposition 2 . 
We use Theorem 3 and Lemma 5 to get the following corollary.

Corollary 6. $Q_{4}^{k}$ is not a divisor graph if and only if $k=2$.

Lemma 7. $Q_{5}^{3}$ is not a divisor graph.

Proof. The induced subgraph on $\{00001,10000,10110,11111,01011\}$ in $Q_{5}^{3}$ is isomorphic to $C_{5}$. Hence, $Q_{5}^{3}$ is not a divisor graph by Proposition 2 .

By Theorem 3, Lemma 5, Proposition 2, and Lemma 7, we get the following corollary.

Corollary 8. $Q_{5}^{k}$ is not a divisor graph if and only if $k=2$ or 3 .

For any positive integer $n$ greater than three, we have the following lemma.

Lemma 9. For $n \geq 4, Q_{n}^{(n-2)}$ is not a divisor graph.

Proof. The result follows from Lemmas 5 and 7 when $n=4$ and 5. For $n \geq 6$, we look at the induced subgraph, say $G_{1}$, on $S=\left\{a_{1}, a_{2}, a_{3}, a_{4}, a_{5}, a_{6}\right\}$ in $Q_{n}^{(n-2)}$, where the $a_{i^{\prime} s}$ are:

$$
\begin{aligned}
& a_{1}=\underbrace{0 \ldots \ldots \ldots \ldots \ldots \ldots \ldots \ldots \ldots \ldots \ldots \ldots \ldots \ldots \ldots \ldots \ldots}_{n}, \\
& a_{2}=\underbrace{0 \ldots \ldots \ldots \ldots \ldots \ldots \ldots \ldots \ldots \ldots \ldots \ldots \ldots}_{n-2} 011 \text {, } \\
& a_{3}=\underbrace{0 \ldots \ldots \ldots \ldots \ldots \ldots \ldots \ldots \ldots \ldots \ldots \ldots \ldots \ldots \ldots \ldots}_{n-3} 01, \\
& a_{4}=\underbrace{1 \ldots \ldots \ldots \ldots \ldots \ldots \ldots \ldots \ldots \ldots \ldots}_{n-3} 1000 \text {, } \\
& a_{5}=\underbrace{1 \ldots \ldots \ldots \ldots \ldots \ldots \ldots \ldots \ldots \ldots \ldots \ldots \ldots \ldots}_{n-3} 1,
\end{aligned}
$$

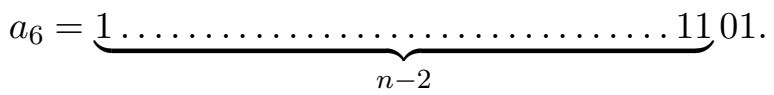

The graph $G_{1}$ is represented in Figure 2. Assume to the contrary that $G_{1}$ is a divisor graph. Hence, it has a divisor orientation, say $D$. Suppose that $\left(a_{1}, a_{2}\right) \in$ $E(D)$. Since $a_{1} a_{5} \notin E\left(G_{1}\right)$, we must have $\left(a_{5}, a_{2}\right) \in E(D)$. We get $\left(a_{3}, a_{2}\right) \in$ $E(D)$, because $a_{3} a_{5} \notin E\left(G_{1}\right)$. We must have $\left(a_{1}, a_{4}\right),\left(a_{3}, a_{6}\right) \in E(D)$, because $a_{4} a_{2}, a_{6} a_{2} \notin E\left(G_{1}\right)$.

Now, assume that $\left(a_{4}, a_{6}\right) \in E(D)$, and since $\left(a_{1}, a_{4}\right) \in E(D)$, we get $\left(a_{1}, a_{6}\right) \in E(D)$, which is a contradiction, since $a_{1} a_{6} \notin E\left(G_{1}\right)$. Otherwise, if $\left(a_{6}, a_{4}\right) \in E(D)$, and since $\left(a_{3}, a_{6}\right) \in E(D)$, then we get $\left(a_{3}, a_{4}\right) \in E(D)$. This leads to a contradiction, since $a_{4} a_{3} \notin E\left(G_{1}\right)$. Similarly, we argue the case $\left(a_{2}, a_{1}\right) \in E(D)$. 


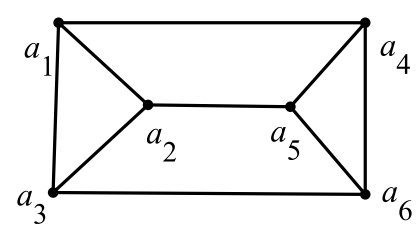

Figure 2. $G_{1}$.

Theorem 10. If $n \geq 5$ and $2 \leq k \leq n-3$, then $Q_{n}^{k}$ is not a divisor graph.

Proof. For any positive integer $k$ with $2 \leq k \leq n-3$, we have the following nested induced subgraphs: $Q_{n}^{k} \supseteq \cdots \supseteq Q_{k+3}^{k} \supseteq Q_{k+2}^{k}$. But, $Q_{k+2}^{k}$ is not a divisor graph, by previous lemma. Hence, $Q_{n}^{k}$ is not a divisor graph, by Proposition 2.

Using the previous theorem, Lemma 9, and Theorem 3, we get the following corollary.

Corollary 11. Suppose $n \geq 4$ and $k \geq 2$. Then $Q_{n}^{k}$ is a divisor graph if and only if $k \geq n-1$.

\section{Characterizing when Powers of Folded-Hypercubes Are Divisor GRAPHS}

In this section we characterize when powers of folded-hypercubes are divisor graphs. First we give the definition of the folded-hypercube.

Definition. A folded-hypercube, denoted by $F Q_{n}$ is a graph whose vertex set is $V\left(Q_{n}\right)$ and two vertices $x$ and $y$ are adjacent if $d_{Q_{n}}(x, y)=1$ or $n$, i.e., $x$ and $y$ differ in exactly one or $n$ bits.

A folded-hypercube is a standard hypercube with some extra edges between the vertices with complementary binary strings. In addition, if $n$ is odd then $F Q_{n}$ is bipartite, see [9]. Hence, $F Q_{n}$ is a divisor graph if $n$ is odd or $n=2$. While for $n \geq 4$ is even, we get the following lemma.

Lemma 12. If $n$ is an even integer with $n \geq 4$, then $F Q_{n}$ is not a divisor graph.

Proof. Consider the set $A=\left\{a_{1}, a_{2}, \ldots, a_{n+1}\right\}$ in $V\left(F Q_{n}\right)$, where

$$
\begin{aligned}
& a_{1}=0 \underbrace{1 \ldots \ldots \ldots \ldots \ldots \ldots \ldots \ldots \ldots \ldots \ldots \ldots 1}, \\
& a_{2}=00 \underbrace{1 \ldots \ldots \ldots \ldots \ldots \ldots \ldots \ldots \ldots \ldots 1}_{n-2},
\end{aligned}
$$




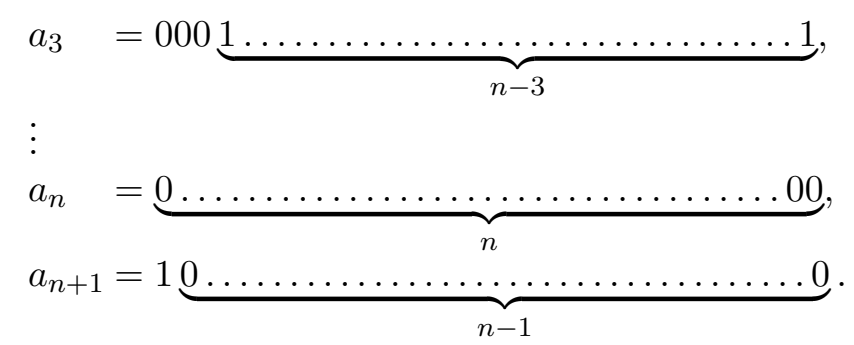

The induced subgraph on $A$ in $F Q_{n}$ is isomorphic to $C_{n+1}$, which is an odd cycle. Hence, $F Q_{n}$ is not a divisor graph by Proposition 2.

We denote the complement of $x$ by $\bar{x}$. According to the definition of foldedhypercube, we get $d_{F Q_{n}}(x, y)=\min \left\{d_{Q_{n}}(x, y), d_{Q_{n}}(x, \bar{y})+1\right\}=\min \left\{d_{Q_{n}}(x, y)\right.$, $\left.d_{Q_{n}}(\bar{x}, y)+1\right\}$. This gives that $\left(F Q_{n}\right)^{\left\lceil\frac{n}{2}\right\rceil^{n}}=K_{2^{n}}$, i.e., $\left(F Q_{n}\right)^{\left\lceil\frac{n}{2}\right\rceil}$ is a divisor graph. Hence, for $n=2,3,4$ and $k \geq 2$, we get $\left(F Q_{n}\right)^{k}$ is a divisor graph. For $n \geq 5$, we get the following results.

Lemma 13. $\left(F Q_{n}\right)^{2}$ is not a divisor graph, for $n \geq 5$.

Proof. Consider the induced subgraph on the set $S=\left\{a_{1}, a_{2}, a_{3}, a_{4}, a_{5}, a_{6}\right\}$ in $\left(F Q_{n}\right)^{2}$, where the $a_{i^{\prime} s}$ are:

$$
\begin{aligned}
& a_{1}=\underbrace{0 \ldots \ldots \ldots \ldots \ldots \ldots \ldots \ldots \ldots \ldots \ldots \ldots \ldots \ldots \ldots}_{n-5} 00000 \text {, } \\
& a_{2}=\underbrace{0 \ldots \ldots \ldots \ldots \ldots \ldots \ldots \ldots \ldots \ldots \ldots \ldots \ldots \ldots \ldots}_{n-5} 11111 \text {, } \\
& a_{3}=\underbrace{0 \ldots \ldots \ldots \ldots \ldots \ldots \ldots \ldots \ldots \ldots \ldots \ldots}_{n-5} 11100 \text {, } \\
& a_{4}=\underbrace{0 \ldots \ldots \ldots \ldots \ldots \ldots \ldots \ldots \ldots \ldots \ldots \ldots}_{n-5} 01110 \text {, }
\end{aligned}
$$

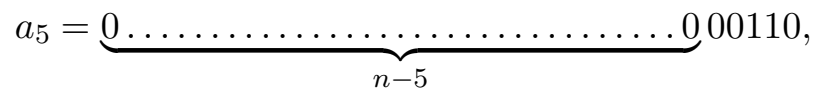

$$
\begin{aligned}
& a_{6}=\underbrace{0 \ldots \ldots \ldots \ldots \ldots \ldots \ldots \ldots \ldots \ldots \ldots \ldots}_{n-5} 011000 .
\end{aligned}
$$

The induced subgraph on the set $S$ in $\left(F Q_{5}\right)^{2}$ is given in Figure 3. This induced subgraph is isomorphic to $G_{1}$ and the result follows by Lemma 9 .

For $n \geq 6$, the induced subgraph on the set $S_{1}=\left\{a_{1}, a_{3}, a_{4}, a_{5}, a_{6}\right\}$ in $\left(F Q_{n}\right)^{2}$ is isomorphic to $C_{5}$. The result follows by Proposition 2 .

Lemma 14. $\left(F Q_{n}\right)^{3}$ is not a divisor graph, for $n \geq 7$. 


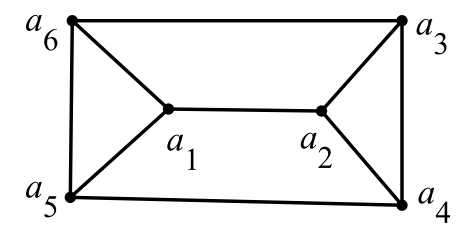

Figure 3. The induced subgraph on $S$.

Proof. Consider the induced subgraph on the set $S=\left\{a_{1}, a_{2}, a_{3}, a_{4}, a_{5}\right\}$ in $\left(F Q_{n}\right)^{3}$, where the $a_{i^{\prime} s}$ are:

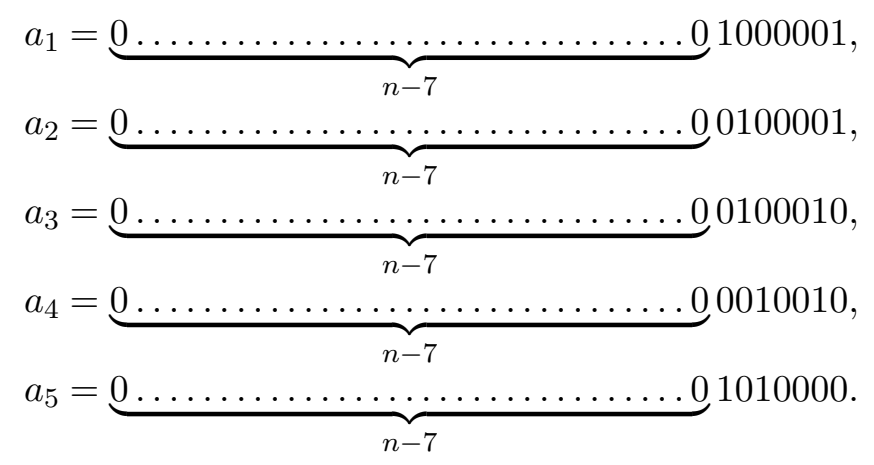

For $n \geq 7$, the induced subgraph on the set $S$ in $\left(F Q_{n}\right)^{3}$ is isomorphic to $C_{5}$. The result follows by Proposition 2.

For $k=4,5$ with $n \geq 2 k+1$, we get that $\left(F Q_{n}\right)^{k}$ is not a divisor graph. The proof is similar to that of Lemma 13 . For $k \geq 6$, we proceed as follows.

Lemma 15. Suppose $i=3$ or $4, k=2 i, n_{i}=4 i+1$, and $n \geq n_{i}$. Then $\left(F Q_{n}\right)^{k}$ is not a divisor graph.

Proof. Consider the induced subgraph on the set $S=\left\{a_{1}, a_{2}, a_{3}, a_{4}, a_{5}, a_{6}\right\}$ in $\left(F Q_{n}\right)^{k}$, where the $a_{i^{\prime} s}$ are:

$$
\begin{aligned}
a_{1} & =\underbrace{0 \ldots \ldots 0}_{n-n_{i}} 1 \underbrace{0 \ldots 00}_{i} 1 \underbrace{0 \ldots 00}_{i} \underbrace{1 \ldots 11}_{i} \underbrace{0 \ldots 00}_{i-1}, \\
a_{2} & =\underbrace{0 \ldots \ldots}_{n-n_{i}} \underbrace{1 \ldots \ldots}_{i+2} \underbrace{0 \ldots 0}_{i} \underbrace{1 \ldots \ldots \ldots 11}_{2 i-1}, \\
a_{3} & =\underbrace{0 \ldots \ldots}_{n-n_{i}} \underbrace{0 \ldots 0}_{i-1} \underbrace{0 \ldots 1 \ldots 1}_{i+1} 0011 \underbrace{0 \ldots 0}_{i-2} 11 \underbrace{0 \ldots 0}_{i-3}, \\
a_{4} & =\underbrace{0 \ldots \ldots 0}_{n-n_{i}} 1 \underbrace{00 \ldots 0}_{i-2} 111 \underbrace{00 \ldots 0}_{i-2} 1100 \underbrace{1 \ldots 1}_{2 i-4} 0, \text { for } i=3,
\end{aligned}
$$




$$
\begin{aligned}
& a_{4}=\underbrace{0 \ldots \ldots 0}_{n-n_{i}} 1 \underbrace{00 \ldots 0}_{i-2} 111 \underbrace{00 \ldots 0}_{i-2} 1100 \underbrace{1 \ldots 1}_{2 i-4} 1, \text { for } i=4, \\
& a_{5}=\underbrace{0 \ldots \ldots}_{n-n_{i}} \underbrace{00 \ldots 0}_{i-1} 11100 \underbrace{11 \ldots 1}_{i-2} 00 \underbrace{1 \ldots 1}_{2 i-5} 00, \\
& a_{6}=\underbrace{0 \ldots \ldots}_{n-n_{i}} 00 \underbrace{1 \ldots \ldots}_{i+2} \underbrace{00 \ldots \ldots 0}_{2 i-4} 1 \underbrace{00 \ldots 0}_{i-1} 1 .
\end{aligned}
$$

The induced subgraph on the set $S$ in $\left(F Q_{n_{i}}\right)^{k}$ is isomorphic to the graph in Figure 3. This induced subgraph is isomorphic to $G_{1}$ and the result follows by Lemma 9. For $n \geq 1+n_{i}$, the induced subgraph on the set $S_{1}=\left\{a_{2}, a_{3}, a_{4}, a_{5}, a_{6}\right\}$ in $\left(F Q_{n}\right)^{k}$ is isomorphic to $C_{5}$. The result follows by Proposition 2.

Lemma 16. Suppose $i=3$ or $4, k=2 i+1, n_{i}=4 i+3$, and $n \geq n_{i}$. Then $\left(F Q_{n}\right)^{k}$ is not a divisor graph.

Proof. Consider the induced subgraph on the set $S=\left\{a_{1}, a_{2}, a_{3}, a_{4}, a_{5}\right\}$ in $\left(F Q_{n}\right)^{k}$, where the $a_{i^{\prime} s}$ are:

$$
\begin{aligned}
a_{1} & =\underbrace{0 \ldots \ldots 0}_{n-n_{i}} 1 \underbrace{0 \ldots 00}_{i} 1 \underbrace{0 \ldots 00}_{i} \underbrace{1 \ldots 1}_{i} \underbrace{0 \ldots 0}_{i-1} 11, \\
a_{2} & =\underbrace{0 \ldots \ldots}_{n-n_{i}} 10 \underbrace{1 \ldots 1}_{i} \underbrace{0 \ldots 0}_{i} \underbrace{1 \ldots \ldots 11}_{2 i-1} 00, \\
a_{3} & =\underbrace{0 \ldots \ldots 0}_{n-n_{i}} \underbrace{0 \ldots 0}_{i-1} \underbrace{1 \ldots 1}_{i+1} 0011 \underbrace{0 \ldots 0}_{i-2} 1 \underbrace{0 \ldots 0}_{i-2} 1, \\
a_{4} & =\underbrace{0 \ldots \ldots}_{n-n_{i}} 00 \underbrace{1 \ldots \ldots}_{i+2} \underbrace{0 \ldots 00}_{2 i-4} 1 \underbrace{0 \ldots 0}_{i-1} 111, \\
a_{5} & =\underbrace{0 \ldots \ldots}_{n-n_{i}} \underbrace{0 \ldots 0}_{i-1} 11100 \underbrace{1 \ldots 11}_{i-2} 00 \underbrace{1 \ldots 11}_{2 i-5} 0011 .
\end{aligned}
$$

For $n \geq n_{i}$, the induced subgraph on the set $S$ in $\left(F Q_{n}\right)^{k}$ is isomorphic to $C_{5}$. The result follows by Proposition 2 .

Theorem 17. Suppose $i \geq 5, k=2 i, n_{i}=4 i+1$, and $n \geq n_{i}$. Then $\left(F Q_{n}\right)^{k}$ is not a divisor graph.

Proof. Consider the induced subgraph on the set $S=\left\{a_{1}, a_{2}, a_{3}, a_{4}, a_{5}, a_{6}\right\}$ in $\left(F Q_{n}\right)^{k}$, where the $a_{i^{\prime} s}$ are:

$$
\begin{aligned}
& a_{1}=\underbrace{0 \ldots 0}_{n-n_{i}} 1 \underbrace{0 \ldots \ldots \ldots 0}_{i} 1 \underbrace{0 \ldots \ldots 00}_{i} \underbrace{1 \ldots \ldots \ldots 1}_{i} \underbrace{00 \ldots \ldots \ldots 00}_{i-1}, \\
& a_{2}=\underbrace{0 \ldots 0}_{n-n_{i}} 1 \underbrace{1 \ldots \ldots \ldots 1}_{i} 1 \underbrace{0 \ldots \ldots 00}_{i} \underbrace{1 \ldots \ldots \ldots 1}_{i} \underbrace{11 \ldots \ldots \ldots 1}_{i-1}, \\
& a_{3}=\underbrace{0 \ldots 0}_{n-n_{i}} \underbrace{0 \ldots 0}_{\left\lfloor\frac{i}{2}\right\rfloor+1} \underbrace{1 \ldots 1}_{\left\lceil\frac{i}{2}\right\rceil} 1 \underbrace{1 \ldots 1}_{\left\lfloor\frac{i}{2}\right\rfloor} \underbrace{0 \ldots 0}_{\left\lceil\frac{i}{2}\right\rceil} \underbrace{1 \ldots 1}_{\left\lceil\frac{i}{2}\right\rceil} \underbrace{0 \ldots 0}_{\left\lfloor\frac{i}{2}\right\rfloor} \underbrace{1 \ldots 11}_{\left\lceil\frac{i-1}{2}\right\rceil} \underbrace{00 \ldots 00}_{\left\lfloor\frac{i-1}{2}\right\rfloor-1} 1,
\end{aligned}
$$


for $i$ odd,

$$
a_{3}=\underbrace{0 \ldots 0}_{n-n_{i}} 0 \underbrace{0 \ldots 0}_{\left\lfloor\frac{i}{2}\right\rfloor} \underbrace{1 \ldots 1}_{\left\lceil\frac{i}{2}\right\rceil} 1 \underbrace{1 \ldots 1}_{\left\lfloor\frac{i}{2}\right\rfloor} \underbrace{0 \ldots 0}_{\left\lceil\frac{i}{2}\right\rceil} \underbrace{1 \ldots 1}_{\left\lceil\frac{i}{2}\right\rceil} \underbrace{0 \ldots 0}_{\left\lfloor\frac{i}{2}\right\rfloor} \underbrace{1 \ldots 1}_{\left\lceil\frac{i-1}{2}\right\rceil} \underbrace{0 \ldots 0}_{\left\lfloor\frac{i-1}{2}\right\rfloor},
$$

for $i$ even,

$$
a_{4}=\underbrace{0 \ldots 0}_{n-n_{i}} 1 \underbrace{0 \ldots 0}_{\left\lfloor\frac{i}{2}\right\rfloor} \underbrace{1 \ldots 1}_{\left\lceil\frac{i}{2}\right\rceil} 1 \underbrace{0 \ldots 0}_{\left\lfloor\frac{i}{2}\right\rfloor} \underbrace{1 \ldots 1}_{\left\lceil\frac{i}{2}\right\rceil} \underbrace{0 \ldots 0}_{\left\lceil\frac{i}{2}\right\rceil} \underbrace{1 \ldots 1}_{\left\lfloor\frac{i}{2}\right\rfloor} \underbrace{1 \ldots 1}_{\left\lceil\frac{i-1}{2}\right\rceil} \underbrace{0 \ldots 0}_{\left\lfloor\frac{i-1}{2}\right\rfloor},
$$

for $i$ odd,

$$
a_{4}=\underbrace{0 \ldots 0}_{n-n_{i}} 1 \underbrace{0 \ldots 0}_{\left\lfloor\frac{i}{2}\right\rfloor} \underbrace{1 \ldots 1}_{\left\lceil\frac{i}{2}\right\rceil} 1 \underbrace{0 \ldots 0}_{\left\lfloor\frac{i}{2}\right\rfloor} \underbrace{1 \ldots 1}_{\left\lceil\frac{i}{2}\right\rceil} \underbrace{0 \ldots 0}_{\left\lceil\frac{i}{2}\right\rceil} \underbrace{1 \ldots 1}_{\left\lfloor\frac{i}{2}\right\rfloor} \underbrace{1 \ldots 1}_{\left\lceil\frac{i-1}{2}\right\rceil\left\lfloor\frac{i-1}{2}\right\rfloor-1} \underbrace{0 \ldots 00} 1,
$$

for $i$ even,

$$
\begin{aligned}
& a_{5}=\underbrace{0 \ldots 0}_{n-n_{i}} 0 \underbrace{0 \ldots 0}_{\left\lfloor\frac{i}{2}\right\rfloor} \underbrace{1 \ldots 1}_{\left\lceil\frac{i}{2}\right\rceil} 1 \underbrace{0 \ldots 0}_{\left\lceil\frac{i}{2}\right\rceil} \underbrace{1 \ldots 1}_{\left\lfloor\frac{i}{2}\right\rfloor} \underbrace{0 \ldots 0}_{\left\lceil\frac{i}{2}\right\rceil} \underbrace{1 \ldots 11}_{\left\lfloor\frac{i}{2}\right\rfloor-1} \underbrace{1 \ldots 1}_{\left\lceil\frac{i-1}{2}\right\rceil} \underbrace{00 \ldots 00}_{\left\lfloor\frac{i-1}{2}\right\rfloor+1}, \\
& a_{6}=\underbrace{0 \ldots 0}_{n-n_{i}} 0 \underbrace{0 \ldots 0}_{\left\lfloor\frac{i}{2}\right\rfloor-1} \underbrace{1 \ldots 1}_{\left\lceil\frac{i}{2}\right\rceil+1} 0 \underbrace{1 \ldots 1}_{\left\lceil\frac{i}{2}\right\rceil} \underbrace{0 \ldots 0}_{\left\lfloor\frac{i}{2}\right\rfloor} \underbrace{1 \ldots 1}_{\left(\left\lceil\frac{i}{2}\right\rceil\right)-2} \underbrace{0 \ldots 0}_{\left\lfloor\frac{i}{2}\right\rfloor} \underbrace{1 \ldots 1}_{\left\lceil\frac{i-1}{2}\right\rceil} \underbrace{0 \ldots 00}_{\left\lfloor\frac{i-1}{2}\right\rfloor+1} 1 .
\end{aligned}
$$

The induced subgraph on the set $S$ in $\left(F Q_{n_{i}}\right)^{k}$ is isomorphic to the graph in Figure 3. This induced subgraph is isomorphic to $G_{1}$ and the result follows by Lemma 9. For $n \geq 1+n_{i}$, the induced subgraph on the set $S_{1}=\left\{a_{2}, a_{3}, a_{4}, a_{5}, a_{6}\right\}$ in $\left(F Q_{n}\right)^{k}$ is isomorphic to $C_{5}$. The result follows by Proposition 2 .

Theorem 18. Suppose $i \geq 5, k=2 i+1, n_{i}=4 i+3$, and $n \geq n_{i}$. Then $\left(F Q_{n}\right)^{k}$ is not a divisor graph.

Proof. Consider the induced subgraph on the set $S=\left\{a_{1}, a_{2}, a_{3}, a_{4}, a_{5}\right\}$ in $\left(F Q_{n}\right)^{k}$, where the $a_{i^{\prime} s}$ are:

$$
\begin{aligned}
& a_{1}=\underbrace{0 \ldots 0}_{n-n_{i}} 1 \underbrace{0 \ldots \ldots \ldots 00}_{i} 1 \underbrace{0 \ldots \ldots \ldots 0}_{i} \underbrace{1 \ldots \ldots \ldots 1}_{i} \underbrace{0 \ldots \ldots \ldots 00}_{i-1} 11, \\
& a_{2}=\underbrace{0 \ldots 0}_{n-n_{i}} 10 \underbrace{1 \ldots \ldots \ldots 1}_{i-1} 1 \underbrace{0 \ldots \ldots \ldots 0}_{i} \underbrace{1 \ldots \ldots \ldots 1}_{i} \underbrace{1 \ldots \ldots \ldots 11}_{i-1} 00 \text {, } \\
& a_{3}=\underbrace{0 \ldots 0}_{n-n_{i}} 0 \underbrace{0 \ldots 0}_{\left\lfloor\frac{i}{2}\right\rfloor} \underbrace{1 \ldots 1}_{\left\lceil\frac{i}{2}\right\rceil} 1 \underbrace{1 \ldots 1}_{\left\lfloor\frac{i}{2}\right\rfloor} \underbrace{0 \ldots 0}_{\left\lceil\frac{i}{2}\right\rceil} \underbrace{1 \ldots 1}_{\left\lceil\frac{i}{2}\right\rceil} \underbrace{0 \ldots 0}_{\left\lfloor\frac{i}{2}\right\rfloor} \underbrace{1 \ldots 1}_{\left\lceil\frac{i-1}{2}\right\rceil} \underbrace{0 \ldots 0}_{\left\lfloor\frac{i-1}{2}\right\rfloor-1} 101,
\end{aligned}
$$

for $i$ odd,

$$
a_{3}=\underbrace{0 \ldots 0}_{n-n_{i}} 0 \underbrace{0 \ldots 0}_{\left\lfloor\frac{i}{2}\right\rfloor} \underbrace{1 \ldots 1}_{\left\lceil\frac{i}{2}\right\rceil} 1 \underbrace{1 \ldots 1}_{\left\lfloor\frac{i}{2}\right\rfloor} \underbrace{0 \ldots 0}_{\left\lceil\frac{i}{2}\right\rceil} \underbrace{1 \ldots 1}_{\left\lceil\frac{i}{2}\right\rceil} \underbrace{0 \ldots 0}_{\left\lfloor\frac{i}{2}\right\rfloor} \underbrace{1 \ldots 1}_{\left\lceil\frac{i-1}{2}\right\rceil} \underbrace{0 \ldots 0}_{\left\lfloor\frac{i-1}{2}\right\rfloor} 01,
$$


for $i$ even,

$$
\begin{aligned}
& a_{4}=\underbrace{0 \ldots 0}_{n-n_{i}} \underbrace{0 \ldots 0}_{\left\lfloor\frac{i}{2}\right\rfloor} \underbrace{1 \ldots 1}_{\left\lceil\frac{i}{2}\right\rceil+1} 0 \underbrace{1 \ldots 1}_{\left\lceil\frac{i}{2}\right\rceil} \underbrace{0 \ldots 0}_{\left\lfloor\frac{i}{2}\right\rfloor} \underbrace{1 \ldots 1}_{\left\lceil\frac{i}{2}\right\rceil-2} \underbrace{0 \ldots 0}_{\left\lfloor\frac{i}{2}\right\rfloor} \underbrace{1 \ldots 1}_{\left\lceil\frac{i-1}{2}\right\rceil} \underbrace{0 \ldots 00}_{\left\lfloor\frac{i-1}{2}\right\rfloor+1} 111, \\
& a_{5}=\underbrace{0 \ldots 0}_{n-n_{i}} 0 \underbrace{0 \ldots 0}_{\left\lfloor\frac{i}{2}\right\rfloor} \underbrace{1 \ldots 1}_{\left\lceil\frac{i}{2}\right\rceil} 1 \underbrace{0 \ldots 0}_{\left\lceil\frac{i}{2}\right\rceil} \underbrace{1 \ldots 1}_{\left\lfloor\frac{i}{2}\right\rfloor} \underbrace{0 \ldots 0}_{\left\lceil\frac{i}{2}\right\rceil} \underbrace{1 \ldots 1}_{\left\lfloor\frac{i}{2}\right\rfloor-1} \underbrace{1 \ldots 1}_{\left\lceil\frac{i-1}{2}\right\rceil} \underbrace{0 \ldots 00}_{\left\lfloor\frac{i-1}{2}\right\rfloor+1} 11 .
\end{aligned}
$$

For $n \geq n_{i}$, the induced subgraph on the set $S$ in $F\left(Q_{n}\right)^{k}$ is isomorphic to $C_{5}$. The result follows by Proposition 2.

Previous results characterize which powers of folded-hypercubes are divisor graphs. We collect these results in the following theorem.

Theorem 19. $\left(F Q_{n}\right)^{k}$ is not a divisor graph iff $2 \leq k \leq\left\lceil\frac{n}{2}\right\rceil-1$, where $n \geq 5$.

\section{REFERENCES}

[1] E.A. AbuHijleh, O.A. AbuGhneim and H. Al-Ezeh, Characterizing when powers of a caterpillar are divisor graphs, Ars Combin. 113 (2014) 85-95.

[2] S. Aladdasi, O.A. AbuGhneim and H. Al-Ezeh, Divisor orientations of powers of paths and powers of cycles, Ars Combin. 94 (2010) 371-380.

[3] S. Aladdasi, O.A. AbuGhneim and H. Al-Ezeh, Characterizing powers of cycles that are divisor graphs, Ars Combin. 97 (2010) 447-451.

[4] S. Al-Addasi, O.A. AbuGhneim and H. Al-Ezeh, Merger and vertex splitting in divisor graphs, Int. Math. Forum 5 (2010) 1861-1869.

[5] S. Aladdasi, O.A. AbuGhneim and H. Al-Ezeh, Further new properties of divisor graphs, J. Combin. Math. Combin. Comput. 81 (2012) 261-272.

[6] G. Agnarsson, R. Greenlaw, Graph Theory: Modeling Applications and Algorithms (Pearson, NJ, USA, 2007).

[7] G. Chartrand, R. Muntean, V. Seanpholphat and P. Zang, Which graphs are divisor graphs, Congr. Numer. 151 (2001) 180-200.

[8] P. Erdős, R. Frued and N. Hegyvári, Arithmetical properties of permutations of integers, Acta Math. Hungar. 41 (1983) 169-176. doi:10.1007/BF01994075

[9] S.Y. Hsieh, C.N. Kuo, Hamiltonian-connectivity and strongly Hamiltonian-laceability of folded hypercubes, Comput. Math. Appl. 53 (2006) 1040-1044. doi:10.1016/j.camwa.2006.10.033

[10] M. Kobeissi, M. Mollard, Disjoint cycles and spanning graphs of hypercubes, Discrete Math. 288 (2004) 73-87.

doi:10.1016/j.disc.2004.08.005 
Which Powers of Hypercubes And Folded Hypercubes Are Divisor ...311

[11] O. Melnikov, V. Sarvanov, R. Tyshkevich, V. Yemelichev and I. Zverovich, Exercises in Graph Theory (Netherlands, Kluwer Academic Publishers, 1998).

doi:10.1007/978-94-017-1514-0

[12] A.D. Pollington, There is a long path in the divisor graph, Ars Combin. 16-B (1983) 303-304.

[13] C. Pomerance, On the longest simple path in the divisor graph, Congr. Numer. 40 (1983) 291-304.

[14] G.S. Singh, G. Santhosh, Divisor graph-I, preprint.

Received 16 January 2014

Revised 22 July 2014

Accepted 22 July 2014 